\title{
Desempenho dos serviços de saúde do SUS de uma macrorregião do estado de Pernambuco, Brasil
}

\author{
Performance of SUS health services in a macroregion of the state of \\ Pernambuco, Brazil
}

Alaine Santos Parente ${ }^{\mathbf{1}}$, Arianny Soares Ramos de Santana ${ }^{\mathbf{1}}$, Sydia Rosana de Araujo Oliveira ${ }^{\mathbf{1}}$

DOI: $10.1590 / 0103-1104202112904$

\begin{abstract}
RESUMO O objetivo deste estudo foi avaliar o desempenho dos serviços de saúde de uma macrorregião do estado de Pernambuco. A pesquisa foi realizada utilizando dados do Projeto de Avaliação do Desempenho do Sistema de Saúde nas dimensões efetividade, acesso, adequação e aceitabilidade. O período de análise foi de 2008 a 2017. O julgamento foi realizado a partir da tendência esperada dos indicadores. Classificouse o desempenho em: excelente ( $\geq 75 \%$ ), satisfatório (50\%-74,9\%), insatisfatório (49\%-25\%) e crítico $(<25 \%)$. O desempenho foi insatisfatório nas dimensões efetividade (41,7\%) e acesso (46\%), e excelente nas dimensões adequação (76,2\%) e aceitabilidade (100\%). Entre os indicadores que contribuíram para o baixo desempenho nas primeiras dimensões, destacam-se: internações por pneumonia, incidência da síndrome da imunodeficiência adquirida, sífilis congênita, vacinação em crianças menores de um ano com tetravalente/pentavalente e procedimentos de alta complexidade. A classificação final revelou resultado satisfatório (52,4\%) na Macrorregião de Saúde. Diante dos resultados, torna-se necessária a observação individual dos indicadores, visando ao planejamento de ações que promovam uma melhora nesse panorama. Esta pesquisa contribuiu para uma ampliação da avaliação de desempenho, divulgando a potencialidade do Projeto de Avaliação do Desempenho do Sistema de Saúde para o monitoramento e a análise do sistema de saúde.
\end{abstract}

PALAVRAS-CHAVE Indicadores de serviços. Regionalização. Pesquisa sobre serviços de saúde.

ABSTRACT The aim of this study was to evaluate the performance of health services in a macro-region in the state of Pernambuco. The research was carried out using data from the Health Services Performance Assessment Methodology Project in the dimensions of effectiveness, access, adequacy and acceptability. The analysis period was from 2008 to 2017. The judgment was made based on the expected trend of the indicators. Performance was classified as: excellent ( $\geq 75 \%)$, satisfactory (50\%-74.9\%), unsatisfactory (49\%-25\%) and critical (<25\%). The performance was unsatisfactory in the dimensions effectiveness (41.7\%) and access (46\%), and excellent in the dimensions adequacy (76.2\%) and acceptability (100\%). Among the indicators that contributed to poor performance in the first dimensions, the following stand out: hospitalizations for pneumonia, incidence of acquired immunodeficiency syndrome, congenital syphilis, vaccination in children under one year of age with tetravalent/pentavalent and highly complex procedures. The final classification revealed a satisfactory result (52.4\%) in the Health Macroregion. In view of the results, it is necessary to observe the indicators individually, aiming at planning actions that promote better results. This research contributed to the expansion of performance evaluation, disclosing the potential of the Health Services

1 Fundação Oswaldo Cruz (Fiocruz) - Instituto Aggeu Magalhães (IAM) - Recife (PE), Brasil.

alaine_15@hotmail.com
Performance Assessment Methodology Project for monitoring and analyzing the health system.

KEYWORDS Indicators of health services. Regional health planning. Health services research. 


\section{Introdução}

A definição de desempenho na saúde não é consensual entre os diversos autores. Consiste em um processo que avalia os resultados alcançados com a implementação de políticas e programas, e em uma atividade imprescindível para a prestação de contas e para a colaboração na tomada de decisão por todos os atores envolvidos. $\mathrm{Ou}$ seja, busca promover melhores resultados no sistema de saúde ${ }^{1-5}$. O desempenho é averiguado por meio do alcance de metas e objetivos estabelecidos ${ }^{6}$, sendo utilizado como sinônimo de efetividade, eficiência, cumprimento de objetivos, resultados e qualidade?.

Vários quadros teóricos são observados na literatura, a exemplo do modelo canadense, do modelo australiano e do modelo da Organização para a Cooperação e Desenvolvimento Econômico (OCDE), que avaliam o desempenho por meio de múltiplas dimensões, apresentando, em comum, a avaliação do estado de saúde da população, os determinantes da saúde e o desempenho do sistema de saúde, que compreende a análise da efetividade, segurança, eficiência e do acesso, entre outras dimensões ${ }^{8-10}$. Observamse, ainda, diversos estudos de comparações internacionais do desempenho dos sistemas de saúde, por meio de múltiplos domínios de desempenho, entre eles: estado de saúde da população, determinantes não médicos de saúde, efetividade, eficiência, acesso, adequação, segurança do paciente, continuidade, qualidade, equidade, sustentabilidade e custo ${ }^{11-16}$.

No Brasil, o Projeto de Avaliação do Desempenho do Sistema de Saúde (Proadess) foi elaborado tendo como referência o modelo canadense. O objetivo é contribuir para o monitoramento e a avaliação de desempenho do sistema de saúde brasileiro, por meio de um conjunto de indicadores que refletem quatro dimensões desse desempenho: determinantes sociais, condições de saúde da população, estrutura do sistema e desempenho dos serviços de saúde, sendo este último o principal objeto de avaliação ${ }^{17,18}$.
Apesar da iniciativa, poucos estudos dedicaram-se ao uso do Proadess. Viacava et al. ${ }^{19}$ analisaram os principais indicadores de desempenho dos serviços nas subdimensões de efetividade, acesso, eficiência e adequação, no período de 1998 a 2010, no Brasil. Esses autores observaram comportamentos diferenciados entre os indicadores de desempenho, com melhores resultados nas subdimensões de efetividade e acesso. No estudo de Ugá et al. ${ }^{20}$, que avaliou a efetividade do sistema de saúde brasileiro, a partir de indicadores referentes a condições evitáveis, verificou-se declínio nos cinco indicadores avaliados, sugerindo melhora nessa efetividade. Laguardia et al.21 analisaram indicadores de adequação do cuidado no Brasil, observando mudanças pouco significativas. Se, por um lado, houve melhoria na atenção à gestante, com crescimento no percentual de mulheres com mais de seis consultas de pré-natal, por outro, visualizou-se piora na adequação ao parto, com crescimento nos partos cesáreos. Mais recentemente, Machado et al.22 avaliaram o desempenho dos serviços de saúde de um município paulista de médio porte, no período de 2008 a 2015, observando melhor desempenho nos indicadores de acesso dos serviços hospitalares, e desempenho desfavorável em indicadores de adequação e efetividade.

Além desses estudos, os autores do Proadess têm publicado alguns relatórios e boletins informativos dos indicadores de desempenho dos serviços de saúde no Brasil ${ }^{23}$, contudo, persistem lacunas em relação ao contexto regional. Sabe-se que a orientação regional direciona o sistema de saúde brasileiro, sendo fundamentais estudos que possam subsidiar o planejamento, a tomada de decisão e a organização dos serviços nas regiões de saúde. Nesse sentido, este estudo apresentou o objetivo de avaliar o desempenho dos serviços em uma Macrorregião de Saúde do estado de Pernambuco. 


\section{Material e métodos}

Trata-se de uma pesquisa avaliativa, de abordagem quantitativa, realizada com base no modelo de avaliação de desempenho do Proadess ${ }^{19}$. Escolheu-se esse modelo por ser uma proposta multidimensional, composta por um conjunto de indicadores estabelecidos a partir da disponibilidade de dados pelos sistemas de informação em saúde do Brasil.

O cenário de investigação foi a III Macrorregião de Saúde, situada no sertão do estado de Pernambuco, composta por três regiões de saúde: Arcoverde (VI), Afogados da Ingazeira (X) e Serra Talhada (XI). Formada por 35 municípios, esta macrorregião teve sua população estimada, em 2019, em 855.144 habitantes24.

A coleta dos dados foi realizada no site do Proadess (https://www.proadess.icict.fiocruz. br/index.php?pag=matrs). Coletaram-se 75 indicadores, contudo, foram incluídos na análise apenas os que possuíam dados em um período de 10 anos, totalizando 42 indicadores. O período de análise foi de 2008 a 2017. Verificaram-se os indicadores da dimensão de desempenho dos serviços da matriz do Proadess nas seguintes subdimensões: efetividade, acesso, adequação e aceitabilidade. As demais foram excluídas pela indisponibilidade de dados no período estudado.

A análise dos dados foi realizada em três etapas. Primeiramente, realizou-se a análise de tendência dos indicadores, por meio de regressão linear simples, no software estatístico $\mathrm{R}$, adotando-se um nível de significância de $5 \%$, e também foram calculadas as variações percentuais dos indicadores entre o primeiro e o último ano da série histórica.

Posteriormente, foi elaborada uma matriz de julgamento (quadro 1), estabelecendo pontuações a partir das tendências esperadas de cada indicador, tendo recebido, cada um deles, uma Pontuação Máxima Esperada (PME) de 1 (tendência crescente ou decrescente). Logo, as PME de cada dimensão foram: efetividade (12), acesso (21), adequação (7) e aceitabilidade (2), com valor total de 42. Para os indicadores que não apresentaram tendências lineares significativas $(p>0,05)$, consideraram-se as variações percentuais (V\%), utilizando como ponto de corte para julgamento de acréscimo ou decréscimo os valores maiores ou iguais a $10 \%$. Consideraram-se as metas estabelecidas pelo estado/Ministério da Saúde (MS) quando elas estavam disponíveis para o indicador, sendo incluídas na matriz de julgamento.

Quadro 1. Matriz de julgamento dos indicadores de desempenho dos serviços de saúde nas dimensões efetividade, acesso, adequação e aceitabilidade

\begin{tabular}{|c|c|c|}
\hline \multicolumn{3}{|l|}{ Efetividade } \\
\hline INDICADOR & DESCRIC̣ÃO DOS VALORES & PME \\
\hline $\begin{array}{l}\text { 1- Percentual de usuários internados com diabetes mellitus, que } \\
\text { realizaram amputação não traumática de membros inferiores }\end{array}$ & $\begin{array}{l}\text { Tendência decrescente }=1 \\
\text { Tendência crescente }=0\end{array}$ & 1 \\
\hline $\begin{array}{l}\text { 2- Taxa de internação por asma, padronizada por sexo e idade, por } \\
100 \text { mil habitantes de } 15 \text { anos ou mais }\end{array}$ & $\begin{array}{l}\text { Tendência decrescente }=1 \\
\text { Tendência crescente }=0\end{array}$ & 1 \\
\hline $\begin{array}{l}\text { 3- Percentual de Internações por Condições Sensíveis à Atenção } \\
\text { Primária (ICSAP) }\end{array}$ & $\begin{array}{l}\text { Tendência decrescente }=1 \\
\text { Tendência crescente }=0\end{array}$ & 1 \\
\hline $\begin{array}{l}\text { 4- Taxa de internação por gastroenterite, padronizada por idade e } \\
\text { sexo, por } 100 \text { mil habitantes de } 1 \text { a } 4 \text { anos }\end{array}$ & $\begin{array}{l}\text { Tendência decrescente }=1 \\
\text { Tendência crescente }=0\end{array}$ & 1 \\
\hline $\begin{array}{l}\text { 5- Taxa de internação por insuficiência cardíaca, padronizada por } \\
\text { sexo, por } 100 \text { mil habitantes de } 40 \text { anos ou mais }\end{array}$ & $\begin{array}{l}\text { Tendência decrescente }=1 \\
\text { Tendência crescente }=0\end{array}$ & 1 \\
\hline $\begin{array}{l}\text { 6- Taxa de internação por pneumonia bacteriana, padronizada por } \\
\text { sexo e idade, por } 100 \text { mil habitantes de } 18 \text { anos ou mais }\end{array}$ & $\begin{array}{l}\text { Tendência decrescente }=1 \\
\text { Tendência crescente }=0\end{array}$ & 1 \\
\hline
\end{tabular}




\section{Quadro 1. (cont.)}

\section{Efetividade}

7- Incidência de aids por 100 mil habitantes

8- Incidência de tuberculose por 100 mil habitantes

9- Número de casos novos de sífilis congênita em menores de 1 ano

10- Taxa de mortalidade por diabetes mellitus, padronizada por sexo e idade, por 100 mil habitantes de 20 a 79 anos

11- Taxa de mortalidade por hipertensão, padronizada por idade e sexo, por 100 mil habitantes de 50 a 64 anos

12- Taxa de mortalidade por infecção respiratória aguda, por 100 mil habitantes menores de 5 anos

PME

\section{ACESSO}

1- Percentual da população coberta pela ESF

2- Cobertura populacional estimada pelas equipes de Atenção Básica

3- Percentual da população de 60 anos ou mais vacinada contra influenza

4- Percentual de crianças menores de 1 ano vacinadas com tetravalente/pentavalente

5- Razão entre o número de procedimentos de mamografia em mulheres de 50 a 69 anos e a metade da população de mulheres na mesma faixa etária

6- Razão entre o número de exames citopatológicos em mulheres de 25 a 64 anos e 1/3 da população de mulheres na mesma faixa etária

7- Taxa de angioplastia, padronizada por sexo e idade, por 100 mil habitantes com 20 anos ou mais

8- Taxa bruta de cirurgias de catarata por 100 mil habitantes com 40 anos ou mais

9- Taxa de internação por artroplastia de quadril, padronizada por sexo e idade, por 100 mil habitantes com 60 anos ou mais

10- Taxa de cirurgia para revascularização do miocárdio, padronizada por sexo e idade, por 100 mil habitantes com 20 anos ou mais

11- Percentual de partos hospitalares

12- Percentual de internações cirúrgicas realizadas fora da região de saúde

13- Percentual de internações cirúrgicas realizadas na região de saúde

14- Percentual de mamografias realizadas fora da região de saúde de residência
Tendência decrescente $=1$

Tendência crescente $=0$

Tendência decrescente $=1$

Tendência crescente $=0$

Tendência decrescente $=1$

Tendência crescente $=0$

Tendência decrescente $=1$

Tendência crescente $=0$

Tendência decrescente $=1$

Tendência crescente $=0$

Tendência decrescente $=1$

Tendência crescente $=0$
1

12

Tendência crescente $=1$

Tendência decrescente $=0$

Tendência crescente ou com alcance de 1

meta, em $2017(80 \%)=1$

Tendência decrescente $=0$

Tendência crescente ou com alcance de 1 meta, em $2017(90 \%)=1$

Tendência decrescente $=0$

Tendência crescente ou com alcance de 1 meta, em $2017(95 \%)=1$

Tendência decrescente $=0$

Tendência crescente ou com alcance de 1 meta, em $2017(0,35)=1$

Tendência decrescente $=0$

Tendência crescente ou com alcance de 1 meta, em $2017(0,44)=1$

Tendência decrescente $=0$

Tendência crescente $=1$

Tendência decrescente $=0$

Tendência crescente $=1$

Tendência decrescente $=0$

Tendência crescente $=1$

Tendência decrescente $=0$

Tendência crescente $=1$

Tendência decrescente $=0$

Tendência crescente $=1$

Tendência decrescente $=0$

Tendência decrescente $=1$

Tendência crescente $=0$

Tendência crescente $=1$

Tendência decrescente $=0$

Tendência decrescente $=1$

Tendência crescente $=0$ 
Quadro 1. (cont.)

\section{Efetividade}

15- Percentual de mamografias realizadas na região de saúde de residência

Tendência crescente $=1$

Tendência decrescente $=0$

16- Percentual de partos hospitalares realizados fora da região de

Tendência decrescente $=1$

Tendência crescente $=0$

saúde de residência

Tendência crescente $=1$

Tendência decrescente $=0$

de residência

Tendência decrescente $=1$

18- Percentual de angioplastias realizadas fora da região de saúde de residência

Tendência crescente $=0$

19- Percentual de quimioterapias realizadas fora da região de saúde de residência

Tendência decrescente $=1$

Tendência crescente $=0$

20- Percentual de quimioterapias realizadas na região de saúde de residência

Tendência crescente $=1$

Tendência decrescente $=0$

21- Percentual de radioterapias realizadas fora da região de saúde de residência

Tendência decrescente $=1$

Tendência crescente $=0$

ADEQUAC̣̃̃O

1- Percentual de pacientes com acidente vascular cerebral internados por até sete dias, que realizaram tomografia computadorizada

Tendência crescente $=1$ Tendência decrescente $=0$

2- Percentual de nascidos vivos cujas mães fizeram mais de seis consultas de pré-natal

Tendência crescente $=1$

Tendência decrescente $=0$

3- Razão entre diálise e transplante renal

Tendência decrescente $=1$

Tendência crescente $=0$

4- Taxa de histerectomias, padronizada por idade, por 100 mil muIheres de 20 anos ou mais

Tendência decrescente $=1$

Tendência crescente $=0$

5- Percentual de meningites bacterianas que tiveram confirmação diagnóstica laboratorial

Tendência crescente $=1$

Tendência decrescente $=0$

6- Percentual de partos cesáreos

Tendência decrescente $=1$

Tendência crescente $=0$

7- Tempo médio de permanência hospitalar de pacientes de 60 anos ou mais, por fratura no quadril

Tendência decrescente $=1$

Tendência crescente $=0$

\section{ACEITABILIDADE}

1- Taxa de abandono do tratamento de tuberculose

2- Percentual de idosos não vacinados contra influenza

Tendência decrescente ou com alcance 1 de meta, em $2016(<5 \%)=1$

Tendência crescente $=0$

Tendência decrescente ou com percen- 1 tual $<10 \%$, em $2017=1$

Tendência crescente $=0$

PME

Fonte: Elaboração própria, a partir dos conceitos estabelecidos pelo Proadess ${ }^{23}$ 
A terceira etapa consistiu no cálculo do desempenho de cada dimensão e do desempenho global, utilizando uma regra de três simples: $\Sigma$ pontuação obtida / $\Sigma$ pontuação máxima x 100. Para a avaliação de desempenho global das regiões de saúde, foram somadas as pontuações obtidas das quatro dimensões: $\Sigma$ efetividade $+\Sigma$ acesso $+\Sigma$ adequação $+\Sigma$ aceitabilidade. Classificou-se o desempenho em: excelente ( $\geq 75 \%)$, satisfatório (50\%-74,9\%), insatisfatório ( $49 \%-25 \%)$ e crítico (<25\%). Essa classificação de desempenho foi elaborada pelos autores, tendo em vista que o Proadess não possui uma matriz de julgamento para os indicadores.

\section{Resultados}

Os indicadores de desempenho das regiões pertencentes à III Macrorregião de Saúde estão apresentados pelas seguintes dimensões: efetividade, acesso, adequação e aceitabilidade (tabela 1).

O desempenho na dimensão efetividade foi satisfatório na X região (50\%) e insatisfatório na VI e na XI região (33,3\% e 41,7\%, respectivamente). $\mathrm{Na}$ análise individual, observou-se desempenho satisfatório nos seguintes indicadores: internação por asma, por gastroenterite, por insuficiência cardíaca (VI, X e XI regiões) e Internações por Condições Sensíveis à Atenção Primária (ICSAP) (X e XI regiões), que apresentaram tendências decrescentes. Entretanto, foi observado resultado insatisfatório nas internações por pneumonia e na incidência da Síndrome da Imunodeficiência Adquirida (Aids) (VI, X e XI regiões), na incidência de tuberculose (VI região) e no número de casos novos de sífilis congênita (VI, X e XI regiões), que foram crescentes entre os anos 2008 e 2017 (tabela 1).

Nos indicadores de acesso aos serviços de saúde, o desempenho foi satisfatório na VI
$(52,4 \%)$ e insatisfatório na $\mathrm{X}$ e na XI região ( $47,6 \%$ e $38,1 \%$, respectivamente). Observouse que o desempenho foi satisfatório nas três regiões de saúde, nos seguintes indicadores: percentual da população coberta pela Estratégia Saúde da Família (ESF), cobertura populacional estimada de equipes de atenção básica, vacinação em idosos contra gripe/influenza, razão de mamografias e citopatológicos, taxas de angioplastias e cirurgias de catarata (tabela 1).

Embora os percentuais de alguns procedimentos realizados fora da região de saúde tenham crescido ou se mantido estacionários, ou seja, tenham apresentado desempenho insatisfatório, conforme a matriz de julgamento, ressalta-se que são inferiores aos percentuais de procedimentos do interior da região de saúde, tais como: internações cirúrgicas, mamografias e partos hospitalares. Contudo, destacam-se os percentuais de radioterapias e angioplastias, os quais são $100 \%$ realizados fora da região de saúde de residência dos usuários, nas três regiões, e o percentual de quimioterapias, na X e na XI região. Apesar da região de Arcoverde ter iniciado a oferta de quimioterapias a partir do ano de 2016, o que gerou decréscimo no indicador, ainda prevalece a sua realização fora da região de saúde (tabela 1).

A estratificação do desempenho na dimensão adequação evidenciou resultado excelente na VI região (85,7\%) e satisfatório na X e na XI região de saúde (ambas com 71,4\%). Entretanto, destaca-se o crescimento do percentual de partos cesáreos nas três regiões de saúde, entre os anos 2008 e 2017. Na dimensão aceitabilidade, observaram-se resultados excelentes (100\%) nas três regiões de saúde, apontando declínio na taxa de abandono de tratamento da tuberculose e no percentual de idosos não vacinados contra influenza (tabela 1). 
Tabela 1. Desempenho, segundo os indicadores de efetividade dos serviços de saúde da VI, X e XI regiões, da III Macrorregião de Saúde do estado de Pernambuco, 2008-2017

\begin{tabular}{|c|c|c|c|c|}
\hline Indicador & PME & PO-VI & PO-X & PO-XI \\
\hline \multicolumn{5}{|l|}{ EFETIVIDADE } \\
\hline $\begin{array}{l}\text { 1- Percentual de usuários internados com diabetes mellitus que realizaram } \\
\text { amputação não traumática de membros inferiores }\end{array}$ & 1 & 0 & 0 & 0 \\
\hline 2- Taxa de internação por asma, por 100 mil habitantes de 15 anos ou mais & 1 & 1 & 1 & 1 \\
\hline 3- Percentual de Internações por Condições Sensíveis à Atenção Primária & 1 & 0 & 1 & 1 \\
\hline 4- Taxa de internação por gastroenterite, por 100 mil habitantes de 1 a 4 anos & 1 & 1 & 1 & 1 \\
\hline $\begin{array}{l}\text { 5- Taxa de internação por insuficiência cardíaca, por } 100 \text { mil habitantes de } 40 \\
\text { anos ou mais }\end{array}$ & 1 & 1 & 1 & 1 \\
\hline $\begin{array}{l}\text { 6- Taxa de internação por pneumonia bacteriana, por } 100 \text { mil habitantes de } 18 \\
\text { anos ou mais }\end{array}$ & 1 & 0 & 0 & 0 \\
\hline 7- Incidência de aids por 100 mil habitantes & 1 & 0 & 0 & 0 \\
\hline 8- Incidência de tuberculose por 100 mil habitantes & 1 & 0 & 1 & 1 \\
\hline 9- Número de casos novos de sífilis congênita em menores de 1 ano & 1 & 0 & 0 & 0 \\
\hline 10- Mortalidade por diabetes mellitus & 1 & 0 & 0 & 0 \\
\hline 11- Mortalidade por hipertensão & 1 & 1 & 0 & 0 \\
\hline 12- Mortalidade por infecção respiratória aguda & 1 & 0 & 1 & 0 \\
\hline PO & 12 & 4 & 6 & 5 \\
\hline Desempenho (\%) & & 33,3 & 50 & 41,7 \\
\hline \multicolumn{5}{|l|}{ ACESSO } \\
\hline 1- Percentual da população coberta pela ESF & 1 & 1 & 1 & 1 \\
\hline 2- Cobertura populacional estimada de equipes de atenção básica & 1 & 1 & 1 & 1 \\
\hline 3- Percentual da população de 60 anos ou mais vacinada contra gripe/influenza & 1 & 1 & 1 & 1 \\
\hline $\begin{array}{l}\text { 4- Percentual de crianças menores de } 1 \text { ano vacinadas com tetravalente/penta- } \\
\text { valente }\end{array}$ & 1 & 0 & 0 & 0 \\
\hline $\begin{array}{l}\text { 5- Razão entre o número de procedimentos de mamografia em mulheres de } 50 \\
\text { a } 69 \text { anos e a metade da população de mulheres na mesma faixa etária }\end{array}$ & 1 & 1 & 1 & 1 \\
\hline $\begin{array}{l}\text { 6- Razão entre o número de exames citopatológicos em mulheres de } 25 \text { a } 64 \\
\text { anos e } 1 / 3 \text { da população de mulheres na mesma faixa etária }\end{array}$ & 1 & 1 & 1 & 1 \\
\hline 7- Taxa de angioplastia por 100 mil habitantes com 20 anos ou mais & 1 & 1 & 1 & 1 \\
\hline 8- Taxa de cirurgias de catarata por 100 mil habitantes com 40 anos ou mais & 1 & 1 & 1 & 1 \\
\hline $\begin{array}{l}\text { 9- Taxa de internação por artroplastia de quadril por } 100 \text { mil habitantes com } 60 \\
\text { anos ou mais }\end{array}$ & 1 & 1 & 0 & 1 \\
\hline $\begin{array}{l}\text { 10- Taxa de cirurgia para revascularização do miocárdio por } 100 \text { mil habitantes } \\
\text { com } 20 \text { anos ou mais }\end{array}$ & 1 & 0 & 1 & 0 \\
\hline 11- Percentual de partos hospitalares & 1 & 1 & 0 & 0 \\
\hline 12- Percentual de internações cirúrgicas realizadas fora da região de saúde & 1 & 0 & 0 & 0 \\
\hline 13- Percentual de internações cirúrgicas realizadas na região de saúde & 1 & 0 & 0 & 0 \\
\hline 14- Percentual de mamografias realizadas fora da região de saúde de residência & 1 & 0 & 1 & 0 \\
\hline 15- Percentual de mamografias realizadas na região de saúde de residência & 1 & 0 & 1 & 0 \\
\hline $\begin{array}{l}\text { 16- Percentual de partos hospitalares realizados fora da região de saúde de } \\
\text { residência }\end{array}$ & 1 & 0 & 0 & 0 \\
\hline $\begin{array}{l}\text { 17- Percentual de partos hospitalares realizados na região de saúde de residên- } \\
\text { cia }\end{array}$ & 1 & 0 & 0 & 0 \\
\hline
\end{tabular}


Tabela 1. (cont.)

\begin{tabular}{|c|c|c|c|c|}
\hline Indicador & PME & PO-VI & PO-X & PO-XI \\
\hline 18- Percentual de angioplastias realizadas fora da região de saúde de residência & 1 & 0 & 0 & 0 \\
\hline $\begin{array}{l}\text { 19- Percentual de quimioterapias realizadas fora da região de saúde de residên- } \\
\text { cia }\end{array}$ & 1 & 1 & 0 & 0 \\
\hline 20- Percentual de quimioterapias realizadas na região de saúde de residência & 1 & 1 & 0 & 0 \\
\hline 21- Percentual de radioterapias realizadas fora da região de saúde de residência & 1 & 0 & 0 & 0 \\
\hline PO & 21 & 11 & 10 & 8 \\
\hline Desempenho (\%) & & 52,4 & 47,6 & 38,1 \\
\hline \multicolumn{5}{|l|}{ ADEQUAC̣ÃO } \\
\hline $\begin{array}{l}\text { 1- Percentual de pacientes com acidente vascular cerebral internados por até } \\
\text { sete dias, que realizaram tomografia computadorizada }\end{array}$ & 1 & 1 & 1 & 1 \\
\hline $\begin{array}{l}\text { 2- Percentual de nascidos vivos cujas mães fizeram mais de seis consultas de } \\
\text { pré-natal }\end{array}$ & 1 & 1 & 1 & 1 \\
\hline 3- Razão entre diálise e transplante renal & 1 & 1 & 1 & 1 \\
\hline 4- Taxa de histerectomias por 100 mil mulheres de 20 anos ou mais & 1 & 1 & 1 & 1 \\
\hline $\begin{array}{l}\text { 5- Percentual de meningites bacterianas que tiveram confirmação diagnóstica } \\
\text { laboratorial }\end{array}$ & 1 & 1 & 0 & 0 \\
\hline 6- Percentual de partos cesáreos & 1 & 0 & 0 & 0 \\
\hline $\begin{array}{l}\text { 7- Tempo médio de permanência hospitalar de pacientes de } 60 \text { anos ou mais, } \\
\text { por fratura no quadril }\end{array}$ & 1 & 1 & 1 & 1 \\
\hline PO & 7 & 6 & 5 & 5 \\
\hline Desempenho (\%) & & 85,7 & 71,4 & 71,4 \\
\hline \multicolumn{5}{|l|}{ ACEITABILIDADE } \\
\hline 1- Taxa de abandono do tratamento de tuberculose & 1 & 1 & 1 & 1 \\
\hline 2- Percentual de idosos não vacinados contra influenza & 1 & 1 & 1 & 1 \\
\hline PO & 2 & 2 & 2 & 2 \\
\hline Desempenho (\%) & 100 & 100 & 100 & \\
\hline
\end{tabular}

Fonte: Elaboração própria, a partir da análise dos dados coletados no Proadess ${ }^{\mathbf{2 3}}$.

PME - Pontuação Máxima Esperada; PO - Pontuação Obtida; Satisfatório = 1, Estável e Insatisfatório = 0.

Desempenho geral: Excelente $\geq 75 \%$; Satisfatório 50\%-74,9\%; Insatisfatório 49\%-25\%; Crítico <25\%.

As dimensões que apresentaram melhores desempenhos foram adequação e aceitabilidade. O desempenho foi categorizado como: excelente, nas dimensões adequação (VI região) e aceitabilidade (VI, X e XI regiões); satisfatório, nas dimensões efetividade ( $\mathrm{X}$ região), acesso (VI região) e adequação (X e XI regiões); e insatisfatório, nas dimensões efetividade (VI e $\mathrm{XI}$ regiões) e acesso (X e XI regiões) (tabela 2). 
Tabela 2. Desempenho global dos serviços da III Macrorregião de Saúde do estado de Pernambuco, 2008-2017

\begin{tabular}{lrrrrrrrr}
\hline & & \multicolumn{1}{c}{ VI } & \multicolumn{2}{c}{ X } & \multicolumn{2}{c}{ XI } & III MACRO \\
DIMENSÃO & PME & PO & $\%$ & PO & $\%$ & PO & $\%$ & $\%$ \\
\hline Efetividade & 12 & 4 & 33,3 & 6 & 50 & 5 & 41,7 & 41,7 \\
Acesso & 21 & 11 & 52,4 & 10 & 47,6 & 8 & 38,1 & 46 \\
Adequação & 7 & 6 & 85,7 & 5 & 71,4 & 5 & 71,4 & 76,2 \\
Aceitabilidade & 2 & 2 & 100 & 2 & 100 & 2 & 100 & 100 \\
PO & 42 & 23 & 54,8 & 23 & 54,8 & 20 & 47,6 & 52,4 \\
\hline
\end{tabular}

Fonte: Elaboração própria, a partir da análise dos dados coletados no Proadess $\mathbf{2 3}$.

PME - Pontuação máxima esperada; PO - Pontuação obtida.

DG - Desempenho Geral: Excelente $\geq 75 \%$; Satisfatório 50\%-74,9\%; Insatisfatório 49\%-25\%; Crítico <25\%.

\section{Discussão}

Os resultados demonstraram desempenho geral satisfatório na III Macrorregião de Saúde do estado de Pernambuco (52,4\%), embora este valor encontre-se no limite inferior (entre satisfatório e insatisfatório). O desempenho excelente nas dimensões adequação e aceitabilidade contribuiu para o alcance desse percentual, visto que as dimensões efetividade e acesso apresentaram desempenho insatisfatório em duas regiões de saúde. Estudo anterior $^{19}$, que avaliou o desempenho dos serviços de saúde no Brasil, no período de 1998 a 2010, observou melhores resultados nas subdimensões efetividade e acesso, decorrentes dos avanços nos indicadores referentes à atuação da atenção primária.

Na dimensão efetividade, a VI e a XI regiões apresentaram desempenho insatisfatório, e a $\mathrm{X}$ região se aproximou do limite inferior, com um resultado de $50 \%$. Se, por um lado, houve declínio em internações por condições evitáveis (percentual geral de ICSAP, internação por asma, por gastroenterite e por insuficiência cardíaca), por outro, observou-se crescimento nas internações por pneumonia, na incidência de aids nas três regiões de saúde, na incidência de tuberculose na VI região e nos casos novos de sífilis congênita nas três regiões de saúde, que contribuíram para o baixo desempenho.

Os resultados nos indicadores de internações por condições evitáveis também têm sido observados em diversos estudos, que demonstraram declínio nessas internações, podendo estar associado ao fortalecimento e à expansão da atenção primária' ${ }^{\mathbf{9}, 20,25-28}$. A redução nas internações pode colaborar com a disponibilização de leitos para outras morbidades e com a redução dos custos hospitalares ${ }^{29}$.

Para a manutenção desses resultados e a redução de custos para o sistema de saúde, torna-se crucial discutir os fatores que influenciam tais resultados e o desenvolvimento de ações que visem à qualificação dos profissionais. Avançar no aperfeiçoamento do modelo assistencial da atenção primária no Brasil, a ESF, proporcionará melhores resultados e acesso mais oportuno para a população ${ }^{28}$.

O monitoramento permanente desses indicadores é indispensável, principalmente diante das mudanças no financiamento da atenção primária, instituídas por meio da Portaria $\mathrm{n}^{0} 2.979 / 2019^{30,31}$. A substituição do piso de atenção básica fixo e variável por um pagamento por capitação ponderada, que utiliza em seu cálculo o número de pessoas cadastradas, pode ocasionar efeitos negativos no modelo da ESF. Além da possibilidade de perda de recursos financeiros, a utilização da população cadastrada para efeitos de financiamento significa o rompimento com a universalidade e equidade do SUS $^{32}$.

Em sequência, destaca-se o crescimento da incidência de aids nas três regiões de saúde do presente estudo. Diferentemente desses 
resultados, dados do MS demonstraram uma queda nos casos de aids, desde 2013, passando de 42.934 casos para 37.161, em $2018^{33}$. O enfrentamento à epidemia de aids requer a realização de ações preventivas, de diagnóstico em tempo oportuno e tratamento adequado ${ }^{34}$.

A tendência crescente da tuberculose na VI região de saúde do estado de Pernambuco também é preocupante. No Brasil, ocorreu uma queda de 1,6\%, no período 2008 a 2017. Porém, Pernambuco está entre os estados que apresentaram, em 2017, os maiores coeficientes de incidência (46/100 mil habitantes). Apesar do decréscimo, os resultados são diversos entre as unidades federativas, demonstrando a necessidade de maior empenho dos gestores, visando eliminar a tuberculose como problema de saúde no País ${ }^{35}$.

Em outro estudo, por meio de dados globais, observou-se declínio de 1,8\%, em 2017, comparando-se com o ano anterior. Contudo, os autores apontam que são necessárias ações para melhorar o diagnóstico, tratamento e a prevenção da doença, para que sejam alcançadas as metas globais para 2020-2035

O crescimento dos casos novos de sífilis congênita nas três regiões de saúde de Pernambuco corrobora com dados de outros estudos, demonstrando ser imprescindível o estabelecimento de ações que visem à prevenção e ao tratamento oportuno da doença $\mathrm{a}^{37,38}$. As taxas crescentes de sífilis podem ter ocorrido devido a uma melhor detecção, diante da oferta de testes rápidos na gestação, a partir da instituição da Rede Cegonha ${ }^{22,38}$. Contudo, ressalta-se que este indicador é apontado como um preditor da qualidade do pré-natal, sendo necessário priorizar investimentos que promovam a melhoria da assistência pré-natal, com diagnóstico precoce da sífilis materna e fortalecimento do tratamento para a prevenção da sífilis congênita ${ }^{38}$.

$\mathrm{Na}$ dimensão acesso, a VI região de saúde apresentou desempenho satisfatório, e a X e a XI regiões, desempenho insatisfatório. Entre os indicadores, destacam-se os resultados positivos na cobertura da ESF e da atenção primária, a vacinação contra influenza em idosos, a razão de mamografias e citopatológicos, a taxa de cirurgias de catarata e de angioplastias.

A tendência crescente na cobertura da ESF também foi apontada em outra pesquisa. As macrorregiões e unidades federativas do Brasil apresentaram crescimento nesse indicador, com aumentos mais significativos no Norte, Sudeste e Sul, os quais apresentaram, em 2016, cobertura igual ou superior a $75 \%{ }^{39}$. Pesquisas revelaram crescimento nos demais indicadores, demonstrando ampliação em alguns procedimentos de média e alta complexidades 19,22,40.

No entanto, destacam-se os procedimentos de alta complexidade (angioplastias, radioterapias e quimioterapias), que são realizados predominantemente fora das três regiões de saúde estudadas. Este resultado corrobora com o estudo de Lima et al. ${ }^{41}$, confirmando vazios assistenciais no SUS, na produção de serviços de alta complexidade no Brasil. Os serviços encontram-se concentrados nas regiões que possuem a capital como município-polo, sobretudo no Nordeste, Norte e Centro-Oeste. Santos $^{\mathbf{4 2}}$ havia descrito que, apesar da existência de 438 regiões de saúde no País, estas não atendem as necessidades da população que está em seu território, referenciando parte dela para outras regiões.

Na dimensão adequação, constatou-se desempenho excelente na VI e satisfatório na $\mathrm{X}$ e na XI região de saúde. Esses resultados decorreram dos achados positivos em seis dos sete indicadores avaliados, observando desempenho insatisfatório nas três regiões apenas no indicador percentual de partos cesáreos, que apresentou crescimento entre os anos 2008 e 2017.

Estudos anteriores haviam apontado crescimento no percentual de partos cesáreos ${ }^{\mathbf{1 9}, 21,22}$. Somente em 2015, estimou-se que 29,7 milhões (21,1\%) dos nascimentos em todo o mundo ocorreram por cesáreas ${ }^{\mathbf{4 3}}$. O Brasil está entre os países que apresentam as mais altas taxas ${ }^{44}$. A Organização Mundial de Saúde (OMS) alertou para a necessidade da realização de partos 
cesáreos somente nos casos indicados, visto que, como qualquer procedimento cirúrgico, pode ocasionar riscos às saúdes materna e infantil, principalmente em mulheres com dificuldades de acesso a assistência obstétrica adequada ${ }^{45}$.

Por fim, a dimensão aceitabilidade apresentou desempenho excelente nas três regiões de saúde. Este resultado deve-se ao declínio nos dois indicadores avaliados: taxa de abandono de tratamento da tuberculose e percentual de idosos não vacinados contra influenza. Mesmo diante dos resultados positivos, aponta-se a necessidade do contínuo monitoramento desses dados e da realização de ações que visem à manutenção de tais resultados. Também é importante destacar que, apesar desse resultado positivo, observou-se crescimento da incidência de tuberculose na VI região de saúde.

Como limitações deste estudo, destacam-se o uso de dados secundários, que podem apresentar erros no processamento e subnotificações, e a inexistência de padrões de desempenho para os indicadores, o que dificultou o seu julgamento. A inexistência de padrões de referência para o julgamento do desempenho também foi exposta no estudo de Reis et al.6.

Apesar das limitações, o modelo do Proadess apresenta grande relevância por disponibilizar aos gestores, pesquisadores e à população um conjunto de indicadores para o monitoramento e a avaliação do sistema de saúde brasileiro, que podem contribuir para a tomada de decisão baseada em evidências e, consequentemente, para o fortalecimento dos serviços e das ações do SUS.

\section{Considerações finais}

Os achados deste trabalho apontam a necessidade de observação individual dos indicadores, visando ao planejamento de ações que promovam melhor desempenho. Também é crucial o monitoramento desses dados, visto que as políticas de austeridade que vêm sendo adotadas no País podem alterar substancialmente esses resultados.

A realização desta pesquisa contribuiu para a ampliação da avaliação de desempenho dos serviços de saúde, utilizando um conjunto de indicadores que refletem, principalmente, o acesso e a efetividade do SUS, divulgando a potencialidade do Proadess para o monitoramento e a avaliação do sistema de saúde brasileiro. Os resultados do estudo podem colaborar com o planejamento, a tomada de decisão e a organização dos serviços nas regiões de saúde.

Sugere-se a realização de novos estudos, que aprofundem a compreensão sobre o desempenho dos serviços, investigando os fatores que podem ter contribuído para melhorar ou piorar o desempenho dessas regiões de saúde. E que avaliem outras dimensões de desempenho propostas pelo Proadess, tais como a análise das condições de saúde e os determinantes sociais, bem como a correlação entre a estrutura do sistema e os resultados do desempenho dos serviços de saúde.

\section{Colaboradoras}

Parente AS (0000-0003-3361-2049)* contribuiu para a concepção e planejamento, análise e interpretação dos dados; elaboração do rascunho e aprovação da versão final do manuscrito. Santana ASR (0000-0003-2772-647X)* contribuiu para a concepção e planejamento; análise e interpretação dos dados e aprovação da versão final do manuscrito. Oliveira SRA (0000-0002-6349-2917)* contribuiu para a concepção e planejamento, análise e interpretação dos dados; revisão crítica do conteúdo e aprovação da versão final do manuscrito. 


\section{Referências}

1. McDavid JC, Huse I, Hawthorn LR. Program Evaluation and Performance Measurement: An Introduction to Practice. 3. ed. Thousand Oaks: Sage Publications; 2018.

2. Papanicolas I, Smith PC. Introduction. In: Papanicolas I, Smith PC, editores. Health system performance comparison: An agenda for policy, information and research. New York: Open University Press; 2013. [acesso em 2020 mar 4]. Disponível em: http://www. euro.who.int/_data/assets/pdf_file/0009/244836/ Health-System-Performance-Comparison.pdf.

3. Smith PC, Mossialos E, Papanicolas I. Performance measurement for health system improvement: experiences, challenges and prospects. Geneva: World Health Organization; 2008. [acesso em 2020 mar 4]. Disponível em: https://www.who.int/management/ district/performance/PerformanceMeasurementHealthSystemImprovement2.pdf.

4. Lizarondo L, Grimmer K, Kumar S. Assisting allied health in performance evaluation: a systematic review. BMC Health Serv Res. 2014 [acesso em 2020 mar 4]; 14:572. Disponível em: https://bmchealthservres.biomedcentral.com/articles/10.1186/s12913-0140572-7.

5. Smith PC, Mossialos E, Papanicolas I, et al. Introduction. In: Smith PC, Mossialos E, Papanicolas I, et al. Performance Measurement for Health System Improvement: Experiences, Challenges and Prospects. Londres: Cambridge University Press; 2009. [acesso em 2020 mar 4]. Disponível em: http://www. euro.who.int/_data/assets/pdf_file/0007/135970/ E94887_Part_I.pdf.

6. Reis AC, Santos EM, Arruda MR, et al. Estudo exploratório dos modelos de avaliação de desempenho em saúde: uma apreciação da capacidade avaliativa. Saúde debate. 2017 [acesso em 2020 mar 4]; 41(esp):330-344. Disponível em: http://www.scielo.br/scielo.php?script=sci_arttext\&pid=S0103$-11042017000500330 \& \operatorname{lng}=e n \& n r m=$ iso\&tlng=pt.
7. Vieira SLM. Avaliação de políticas e programas de saúde. Rio de Janeiro: Editora Fiocruz; 2014.

8. Canadian Institute for Health Information. A Performance Measurement Framework for the Canadian Health System. Ottawa: CIHI; 2013 [acesso em 2019 set 9]. Disponível em: https://secure.cihi.ca/ free_products/HSP_Framework_ Technical_ Report_EN.pdf.

9. Australian Institute of Health Welfare. The National Health Performance Framework. Sidney: AIHW; 2017. [acesso em 2019 set 9]. Disponível em: https:// www.aihw.gov.au/getmedia/0473c334-bb4d-4eca-8fd7-29f15a2ac94f/national-health-performance-framework-figure-31Aug17.pdf.aspx.

10. Carinci F, Gool KV, Mainz J, et al. Towards actionable international comparisons of health system performance: expert revision of the OECD framework and quality indicators. Int J Qual Health Care. 2015 [acesso em 2019 set 9]; 27(2):137-46. Disponível em: https:// academic.oup.com/intqhc/article/27/2/137/1787909.

11. Hejduková P, Kureková L. National health systems' performance: evaluation WHO indicators. Procedia - Social and Behavioral Sciences. 2016 [acesso em 2020 mar 4]; (230):240-248. Disponível em: https://www.sciencedirect.com/science/article/pii/ S1877042816311326.

12. Schneider EC, Sarnak DO, Squires D, et al. Mirror, Mirror 2017: International Comparison Reflects Flaws and Opportunities for Better U.S. Health Care. 2017. [acesso em 2020 abr 25]. Disponível em: https://www.commonwealthfund.org/publications/ fund-reports/2017/jul/mirror-mirror-2017-international-comparison-reflects-flaws-and.

13. Barua B, Hasan S, Timmermans I. Comparing Performance of Universal Health Care Countries. 2017. [acesso em 2020 abr 25]. Disponível em: http://www. fraserinstitute.org.

14. Canadian Institute for Health Information. Interna- 
tional Comparisons at CIHI February 2017. Ottawa: CIHI; 2017. [acesso em 2020 abr 25]. Disponível em: https://secure.cihi.ca/free_products/international-comparisons-en-web.pdf.

15. Conill EM, Xavier DR, Piola SF, et al. Determinantes sociais, condicionantes e desempenho dos serviços de saúde em países da América Latina, Portugal e Espanha. Ciênc. Saúde Colet. 2018 [acesso em 2019 set 9]; 27(3):2171-2185. Disponível em: http://www.scielo.br/scielo.php?pid=S1413$-81232018000702171 \&$ script=sci_abstract\&tlng=pt.

16. Sun D, Ahn H, Lievens T, et al. Evaluation of the performance of national health systems in 2004-2011: An analysis of 173 countries. PLoS One. 2017 [acesso em 2019 set 9]; 12(3). Disponível em: https://doi. org/10.1371/journal.pone.0173346.

17. Viacava F, Almeida C, Caetano R, et al. Uma metodologia de avaliação do desempenho do sistema de saúde brasileiro. Ciênc. Saúde Colet. 2004 [acesso em 2019 set 9]; 9(3):711-724. Disponível em: http:// dx.doi.org/10.1590/S1413-81232004000300021.

18. Viacava F, Laguardia J, Ugá MAD, et al. Proadess: Avaliação de Desempenho do Sistema de Saúde Brasileiro: indicadores para monitoramento. 2012. [acesso em 2020 abr 26]. Disponível em: https://www.proadess. icict.fiocruz.br/index.php?pag=relat.

19. Viacava F, Ugá MAD, Porto S, et al. Avaliação de desempenho de sistemas de saúde: um modelo de análise. Ciênc. Saúde Colet. 2012 [acesso em 2019 set 9]; 17(4):921-934. Disponível em: http://dx.doi. org/10.1590/S1413-81232012000400014.

20. Ugá MAD, Laguardia J, Porto SM, et al. A efetividade do sistema de saúde brasileiro: uma avaliação através de indicadores referentes a condições evitáveis. Reciis. 2013 [acesso em 2019 set 9]; 7(2). Disponível em: http://dx.doi.org/10.3395/reciis.v7i2.495.

21. Laguardia J, Ugá MAD, Porto SM, et al. Análise descritiva da adequação do cuidado no desempenho dos serviços de saúde no Brasil. Tempus Actas de saúde Colet. 2014 [acesso em 2019 set 9]; 8(4). Dis- ponível em: https://www.researchgate.net/publication/307659516.

22. Machado RC, Forster AC, Campos JJB, et al. Avaliação de desempenho dos serviços públicos de saúde de um município paulista de médio porte, Brasil, 2008 a 2015. 2019. [acesso 2020 fev 17]. Disponível em: https://anaisihmt.com/index.php/ihmt/article/ view/319.

23. Fundação Oswaldo Cruz. Metodologia de Avaliação do Desempenho do Sistema de Saúde. Projeto de avaliação de desempenho do sistema de saúde. 2020 [acesso em 2020 abr 25]. Disponível em: https://www. proadess.icict.fiocruz.br/index.php?pag=.

24. Brasil. Ministério da Saúde, Departamento de Informática do SUS (DATASUS). Informações de Saúde. Brasília, DF: Ministério da Saúde; 2020 [acesso em 2020 jan 14]. Disponível em: http://www2.datasus. gov.br/DATASUS/index.php?area=02.

25. Mendonça SS, Albuquerque EC. Perfil das internações por condições sensíveis à atenção primária em Pernambuco, 2008 a 2012. Epidemiol. Serv. Saúde. 2014 [acesso em 2019 set 9]; 23(3):463-474. Disponível em: http://dx.doi.org/10.5123/S167949742014000300009.

26. Ceccon RF, Meneghel SN, Viecili PRN. Internações por condições sensíveis à atenção primária e ampliação da Saúde da Família no Brasil: um estudo ecológico. Rev. bras. epidemiol. 2014 [acesso em 2019 set 9]; 17(4). Disponível em: http://dx.doi.org/10.1590/18094503201400040014 .

27. Gonçalves RF, Souza IMC, Tanaka OY, et al. Programa Mais Médicos no Nordeste: avaliação das internações por condições sensíveis à Atenção Primária à Saúde. Ciênc. Saúde Colet. 2016 [acesso em 2019 set 9]; 21(9):2815-2824. Disponível em: http://dx.doi. org/10.1590/1413-81232015219.15392016.

28. Pinto LF, Giovanella L. Do Programa à Estratégia Saúde da Família: expansão do acesso e redução das internações por condições sensíveis à atenção básica (ICSAB). Ciênc. Saúde Colet. 2018 [acesso em 2020 
fev 17]; 23(6):1903-1913. Disponível em: http://www. scielo.br/pdf/csc/v23n6/1413-8123-csc-23-06-1903. pdf.

29. Torimoto T, Costa JSD. Internações por condições sensíveis à atenção primária, gastos com saúde e Estratégia Saúde da Família: uma análise de tendência. Ciênc. Saúde Colet. 2017 [acesso em 2020 fev 17]; 22(3):1903-1913. Disponível em: http://www. scielo.br/scielo.php?script=sci_arttext\&pid=S1413$-81232017002300891 \& \operatorname{lng}=$ en $\& \mathrm{nrm}=$ iso $\&$ tlng=pt \#B34.

30. Brasil. Ministério da Saúde. Portaria $\mathrm{n}^{\circ} 2.979$, de 12 de novembro de 2019. Institui o Programa Previne Brasil, que estabelece novo modelo de financiamento de custeio da Atenção Primária à Saúde no âmbito do Sistema Único de Saúde, por meio da alteração da Portaria de Consolidação n ${ }^{\circ}$ 6/GM/MS, de 28 de setembro de 2017. Diário Oficial da União. 13 Nov 2019.

31. Massuda A. Mudanças no financiamento da Atenção Primária à Saúde no Sistema de Saúde Brasileiro: avanço ou retrocesso? Ciênc. Saúde Colet. 2020 [acesso em 2020 abr 25]; 25(4). Disponível em: https://doi.org/10.1590/1413-81232020254.01022020.

32. Giovanella L, Franco CM, Almeida PF. Política Nacional de Atenção Básica: para onde vamos? Ciênc. Saúde Colet. 2020 [acesso em 2020 abr 25]; 25(4). Disponível em: https://doi.org/10.1590/141381232020254.01842020 .

33. Brasil. Ministério da Saúde, Secretaria de Vigilância em Saúde. Boletim Epidemiológico HIV Aids 2019. Brasília, DF: Ministério da Saúde; 2019. [acesso em 2020 abr 27]. Disponível em: http://www.aids.gov. br/pt-br/pub/2019/boletim-epidemiologico-de-hivaids-2019.

34. Greco DB. Trinta anos de enfrentamento à epidemia da Aids no Brasil, 1985-2015. Ciênc. Saúde Colet. 2016 [acesso em 2020 abr 25]; 21(5):1553-1564. Disponível em: http://www.scielo.br/scielo.php?script=sci_ arttext\&pid=S1413-81232016000501553\&lng=en\&nr $\mathrm{m}=$ iso\&tlng=pt.
35. Brasil. Ministério da Saúde, Secretaria de Vigilância em Saúde. Implantação do plano nacional pelo fim da tuberculose como problema de saúde pública no Brasil: primeiros passos rumo ao alcance das metas. Boletim Epidemiológico. Brasília, DF: Ministério da Saúde; 2018 [acesso em 2019 set 9]. Disponível em: http://portalarquivos2.saude.gov.br/images/ pdf/2018/marco/26/2018-009.pdf.

36. Macneil A, Glaziou F, Sismanidis C, et al. Global Epidemiology of Tuberculosis and Progress Toward Achieving Global Targets - 2017. Morb Mortal Wkly Rep. 2019 [acesso em 2020 abr 25]; 68(11):263-266. Disponível em: https://www.cdc.gov/mmwr/volumes/68/ wr/mm681la3.htm\#F1_down.

37. Bottura BR, Matuda L, Rodrigues PSC, et al. Perfil epidemiológico da sífilis gestacional e congênita no Brasil - período de 2007 a 2016. Arq Med Hosp Fac Cienc Med Santa Casa São Paulo [internet]. 2019 [acesso em 2020 abr 25]; 64(2):69-75. Disponível em: http://arquivosmedicos.fcmsantacasasp.edu.br/index.php/AMSCSP/article/view/515.

38. Bezerra MLMB, Fernandes FECV, Nunes JPO, et al. Congenital Syphilis as a Measure of Maternal and Child Healthcare, Brazil. Emerging Infectious Diseases. 2019 [acesso em 2020 abr 25]; 25(8). Disponível em: https://wwwnc.cdc.gov/eid/article/25/8/18-0298_article.

39. Neves RG, Duro SMS, Nunes BP, et al. Tendência temporal da cobertura da Estratégia Saúde da Família no Brasil, regiões e Unidades da Federação, 2006-2016. Epidemiol. Serv. Saúde. 2018 [acesso em 2019 set 9]; 27(3). Disponível em: http://dx.doi.org/10.5123/s167949742018000300008.

40. Martins M, Lima SML, Andrade CLT, et al. Indicadores hospitalares de acesso e efetividade e crise econômica: análise baseada nos dados do Sistema Único de Saúde, Brasil e estados da região Sudeste, 2009-2018. Ciênc. Saúde Colet. 2019 [acesso em 2020 abr 25]; 24(12). Disponível em: http://www. scielo.br/scielo.php?script=sci_arttext\&pid=S1413$-81232019001204541 \& \operatorname{lng}=\mathrm{pt} \& \mathrm{nrm}=\mathrm{iso} \& \operatorname{lng}=\mathrm{pt}$. 
41. Lima LD, Albuquerque MV, Scatena JHG, et al. Arranjos regionais de governança do Sistema Único de Saúde: diversidade de prestadores e desigualdade espacial na provisão de serviços. Cad. Saúde Pública. 2019 [acesso em 2019 set 9]; 35(supl2). Disponível em: http://dx.doi.org/10.1590/0102-311x00094618.

42. Santos L. SUS-30 anos: um balanço incômodo? Ciênc. Saúde Colet. 2018 [acesso em 2020 abr 25]; 23(6). Disponível em: http://www.scielo.br/scielo.php?script=sci_arttext \&pid $=$ S1413$-81232018000602043 \& \operatorname{lng}=$ pt\&nrm=iso\&tlng=pt.

43. Boerma T, Ronsmans C, Melesse DY, et al. Global epidemiology of use of and disparities in caesarean sections. The Lancet. 2018 [acesso em 2020 fev 17]; 392(10155):1341-1348. Disponível em: https://www. thelancet.com/journals/lancet/article/PIISO1406736(18)31928-7/fulltext?rss=yes.
44. Betrán AP, Ye J, Moller AB, et al. The Increasing Trend in Caesarean Section Rates: Global, Regional and National Estimates: 1990-2014. PLos One. 2016 [acesso em 2020 fev 17]; 11(2). Disponível em: https://www. ncbi.nlm.nih.gov/pubmed/26849801.

45. Organização Mundial da Saúde. Declaração da OMS sobre Taxas de Cesáreas. Geneva: OMS; 2015. [acesso em 2019 set 9]. Disponível em: https://apps.who. int/iris/bitstream/handle/10665/161442/WHO_ RHR_15.02_por.pdf;jsessionid=CE1B13148F5A4C4 944EACB78A0A3D739? sequence=3.

Recebido em 14/05/2020

Aprovado em 07/12/2020

Conflito de interesses: inexistente

Suporte financeiro: não houve 